\title{
PRODUCCIÓN Y CALIDAD DE UN TOMATE CHERRY EN DOS TIPOS DE INVERNADERO EN CULTIVO SIN SUELO
}

\author{
PRODUCTION AND QUALITY OF CHERRY TOMATOES IN TWO TYPES \\ OF GREENHOUSES IN CULTIVARS WITHOUT SOIL
}

\author{
Pilar Mazuela ${ }^{1}$; Leslie Acuña; Manuel Alvárez; Álvaro Fuentes
}

\section{RESUMEN}

El valle de Azapa, al extremo norte de Chile, posee un clima que le permite producir hortalizas durante todo el año, concentrando la producción durante el invierno. El tomate es el cultivo que más se planta, con una superficie media de 840 hectáreas al aire libre. Muchos agricultores están mejorando los procesos de producción con malla antiinsectos y una minoría está trabajando con invernaderos más sofisticados.

El objetivo del proyecto fue evaluar dos tipos de invernadero, para determinar el efecto sobre la producción y calidad del tomate cherry. Se cultivó en un sistema de cultivo sin suelo, utilizando perlita y compost como sustratos. Los tratamientos fueron: T0: invernadero tipo multitúnel de policarbonato (alta tecnología) y T1: invernadero tipo capilla a un agua, cubierto con malla antiinsectos (baja tecnología). Se cuantificó el efecto del tipo de invernadero sobre los parámetros de fertirriego, producción y la calidad del fruto. En los resultados no se observaron diferencias significativas en la producción, aunque hubo diferencia en el número de frutos, siendo mayor bajo malla antiinsectos. Respecto a la calidad, hubo diferencia significativa en calidad de frutos, con un mayor contenido de sólidos solubles y porcentaje de materia seca en los tomates cultivados en el invernadero de baja tecnología. Con los resultados de estos experimentos se puede concluir que la producción es igual en ambos invernaderos, aunque el tamaño del fruto tiende a ser menor cuando se cultiva bajo malla antiáfido, con un mayor contenido de sólidos solubles y mayor porcentaje de materia seca.

Palabras clave: Cultivos protegidos, Solanum lycopersicum, cultivo sin suelo, malla antiinsectos, cultivo en sustrato.

\section{ABSTRACT}

Azapa valley, in the extreme north of Chile, as a weather that allows to grow vegetables throughout the year but specially during winter. Tomato is the most grown cultivar with an area of 840 hectares. Many farmers are improving the production processes by using an anti-insect web and a few are working with more sophisticated greehouses. The objective of this study was to evaluate two types of greenhouses to determine the effect of them on the production and quality of cherry tomato.

The plants were cultivated in a system without soil, using perlita and compost as sustrate. The treatments were: T0 greenhouse with the form of a tunnel made of polycarbonate (high tech) and T1 greenhouse with the form of a chapel covered with anti-insect webs (low tech). The effect of the type of greenhouse on the watering parameters was quantified, also the production and quality of the fruit. In the results here were not observed significant differences in the production, though there was a difference in the number of fruit, being larger under the anti-insect webs. Regarding the quality, there was a significant difference, with a higher amount of soluble solids and a dry material percentage in the tomatoes grown in the low tech greenhouse. With the results obtained it can be concluded that the production is the same in both greenhouses, but the size of the fruit tends to be smaller when it grows under the web conditions.

Key words: Protected cultivars, Solanum lycopersicum, cultivars without soil, anti-insect web, cultivar in sustrate.

1 Departamento de Producción Agrícola, Facultad de Ciencias Agronómicas, Universidad de Tarapacá, Arica, Chile. Av. General Velásquez 1775. Casilla 6-D. Arica, Chile. Fax: +56 58 220035. E-mail: pmazuela@uta.cl 


\section{INTRODUCCIÓN}

El clima de Arica, en el extremo norte de Chile al límite con Perú, es la principal ventaja de la comuna para la producción de hortalizas al aire libre para consumo en fresco durante todo el año. Sin embargo, dada su ubicación, distante a 2.000 $\mathrm{km}$ de los grandes centros urbanos de Chile, la producción se concentra en hortalizas de alto valor como el tomate y pimiento. La superficie total cultivada con hortalizas es de 2.877 has, donde 2.853 has están al aire libre y 24 has bajo invernadero (INE, 2008). De éstas, la superficie cultivada con tomate alcanza a 840 has. En la región predominan las condiciones de clima de desierto costero con nubosidad abundante, ausencia de heladas, vientos moderados, alta humedad relativa y alta radiación solar directa, durante todo el año. En los últimos años han mejorado las técnicas de cultivo al usar estructuras de protección con malla antiinsectos, con la ventaja de que disminuyen el uso de insecticidas y pueden incorporar el uso de abejorros para la polinización del tomate, logrando un producto de mejor calidad. Möller et al. (2004) indican que los cultivos protegidos reducen la frecuencia de uso de pesticidas, aunque en casos de cultivos protegidos en zonas tropicales las condiciones microclimáticas del invernadero pueden ser menos favorables que al aire libre (Harmanto et al., 2006)

Para las estructuras con malla antiinsectos, Romero-Gómez et al. (2010) indican que su diseño debe considerar un equilibrio entre una buena ventilación y la protección del cultivo dependiendo del enfriamiento deseado y la susceptibilidad del cultivo a los insectos. Diversos autores indican que temperaturas medias entre $21-27^{\circ} \mathrm{C}$ (Abdul-Baki y Stommel, 1995; Sato et al., 2000) y humedad relativa en torno al 60\% (Peet et al., 2003) son óptimas para el cultivo de tomate. Un exceso de temperaturas induce al cierre de estomas y reduce la transpiración y fotosíntesis, en consecuencia, se reduce la tasa de transporte del xilema y la producción de biomasa (Adams y Ho, 1993) que puede implicar una reducción de la producción o calidad del fruto.

\section{MATERIAL Y MÉTODOS}

Para la realización de la investigación los tratamientos consideraron dos tipos de invernadero, uno de alta tecnología tipo multitúnel de policarbonato, automatizado (T0) y otro con baja tecnología, tipo capilla a un agua cubierta con malla antiinsectos (T1). Para optimizar el uso del agua y un mayor control sobre el cultivo se utilizó el sistema de cultivo sin suelo. El material vegetal fue un tomate tipo cereza (cv. 52-71) y los experimentos fueron:

T0: invernadero de alta tecnología

T1: invernadero de baja tecnología

Los parámetros ambientales de temperatura y humedad relativa para ambos invernaderos se describen en el Cuadro 1.

El tomate cherry tuvo un ciclo de inviernoprimavera, el trasplante se realizó el 26 de julio, el inicio de cosecha fue el 16 de noviembre hasta el 18 de diciembre. La densidad de plantación fue de 2,22 plantas por $\mathrm{m}^{-2}$ colocadas en sacos de sustrato de $20 \mathrm{~L}$, con tres plantas por saco. Las plantas fueron conducidas a un eje y se usaron abejorros (Bombus terrestris) para la polinización. La conductividad eléctrica (CE) media del agua de riego fue de $0,8 \mathrm{dS} \mathrm{m}^{-1} ; \mathrm{y} \mathrm{pH} 8,3$. La composición química del agua de riego (en $\mathrm{mmol} \mathrm{L}^{-1}$ ) fue: 0,1 de $\mathrm{NO}_{3}^{-} ; 2,9$ de $\mathrm{HCO}_{3}^{-} ; 2,3$ de $\mathrm{SO}_{4}^{2-} ; 3,3$ de $\mathrm{Cl}^{-}$; 2,8 de $\mathrm{Na}^{+} ; 0,3$ de $\mathrm{K}^{+} ; 3,9$ de $\mathrm{Ca}^{2+}$ y 1,9 de $\mathrm{Mg}^{2+}$. La CE de la disolución nutritiva utilizada fue de 2,3 dS m ${ }^{-1}$ y pH 7,2. La composición media de los nutrientes aportados fue: $13{\mathrm{de} \mathrm{NO}_{3}}^{-} ; 1,75 \mathrm{de}$ $\mathrm{H}_{2} \mathrm{PO}_{4}^{-} ; 2,5$ de $\mathrm{SO}_{4}^{2-} ; 7,5$ de $\mathrm{K}^{+} ; 8$ de $\mathrm{Ca}^{2+}$ y 2,5 de $\mathrm{Mg}^{2+}$, expresados en me $\mathrm{L}^{-1}$.

Los parámetros de fertirriego analizados fueron: conductividad eléctrica $\left(\mathrm{dS} \mathrm{m}^{-1}\right), \mathrm{pH}$, porcentaje de

\section{Cuadro 1}

Temperatura y humedad relativa promedio medias mensuales, según tratamiento: alta tecnología (T0) y baja tecnología (T1)

\begin{tabular}{|l|c|c|c|c|c|c|}
\hline \multicolumn{1}{|c|}{ Mes } & \multicolumn{2}{|c|}{\begin{tabular}{c}
$\mathbf{T}^{\mathbf{0}} \mathbf{\text { máximas }}\left({ }^{\mathbf{0}} \mathbf{C}\right)$ \\
\hline
\end{tabular}} & \multicolumn{2}{c|}{$\mathbf{T}^{\mathbf{0}} \mathbf{\text { mínimas }}\left({ }^{\mathbf{0}} \mathbf{C}\right)$} & \multicolumn{2}{c|}{$\begin{array}{c}\text { Humedad } \\
\text { relativa (\%) }\end{array}$} \\
\hline Julio & 26,6 & 19,3 & 9,8 & 10,1 & 61,6 & 72,2 \\
\hline Agosto & 26,4 & 19,8 & 12,8 & 11,4 & 65,1 & 72,0 \\
\hline Septiembre & 28,9 & 21,0 & 11,7 & 11,4 & 57,7 & 70,1 \\
\hline Octubre & 29,7 & 22,4 & 12,5 & 13,4 & 59,0 & 68,6 \\
\hline Noviembre & 34,8 & 24,1 & 16,2 & 13,9 & 59,3 & 67,0 \\
\hline Diciembre & 36,9 & 27,0 & 15,8 & 15,2 & 60,3 & 66,0 \\
\hline
\end{tabular}


drenaje y consumo hídrico $\left(\mathrm{L} \mathrm{m}^{-2}\right.$ día $\left.{ }^{-1}\right)$. Se evaluó la producción en $\mathrm{kg}$ y número de frutos $\mathrm{m}^{-2}$. Con respecto a la calidad, se cuantificó la firmeza de fruto $(\mathrm{kg})$, los sólidos solubles totales $\left({ }^{\mathrm{o}} \mathrm{Brix}\right) \mathrm{y}$ porcentaje de materia seca. La firmeza de fruto a la presión se determinó con un penetrómetro marca Effegi modelo FT 011 (0-11 lb), utilizando un émbolo de $8 \mathrm{~mm}$ de diámetro. La concentración de sólidos solubles se midió con un refractómetro portátil termocompensado marca Arquimed modelo 2003319. El porcentaje de materia seca se determinó pesando un número establecido de frutos, los que posteriormente fueron secados en la estufa a $60^{\circ} \mathrm{C}$ durantes dos días.

El diseño experimental fue de bloques completamente al azar (Little y Hills, 1976; Petersen, 1994) con dos tratamientos y tres repeticiones. Para la separación de medias se usó la probabilidad asociada a la t de Student. Para los cálculos se utilizó el programa estadístico Microsoft Excel 2000.

\section{RESULTADOS Y DISCUSIÓN}

\section{FERTIRRIEGO}

Los resultados de los parámetros de fertirriego se describen en el Cuadro 2. No se observó diferencia entre los tratamientos para $\mathrm{CE}, \mathrm{pH}$ y porcentaje de drenaje.

\section{PRODUCCIÓN Y CALIDAD DE FRUTOS}

Los parámetros de producción y calidad de frutos se indican en el Cuadro 3. No se observan

Cuadro 2

Parámetros de fertirriego para dos cultivos de tomate cherry, según tipo de invernadero: alta tecnología (T0) y baja tecnología (T1)

\begin{tabular}{|l|c|c|c|}
\cline { 2 - 4 } \multicolumn{1}{c|}{} & $\mathbf{C E}\left(\mathbf{d S} \mathbf{~ m}^{-1}\right)$ & $\mathbf{p H}$ & \% drenaje \\
\hline T0 & 2,86 & 7,51 & 20,59 \\
\hline T1 & 3,06 & 7,55 & 22,97 \\
\hline$p$ & $\mathrm{~ns}$ & $*$ & $\mathrm{~ns}$ \\
\hline
\end{tabular}

$*, * *, * * *$, ns, son $P \leq 0,05, P \leq 0,01, P \leq 0,001$ y no significativo, respectivamente.

CE: conductividad eléctrica. diferencias significativas en la producción de tomate cherry entre tratamientos. Estos resultados coinciden con lo indicado por Max et al. (2009) referido a producción total. Sin embargo, este autor indica que la producción comercial fue mejor en el invernadero de baja tecnología debido a la alta incidencia de frutos afectados por Blossom-end rot (Ber) en el invernadero de mayor tecnología. Respecto a los parámetros de calidad de frutos, los resultados sugieren que el tomate cherry es sensible a los tratamientos, especialmente en contenido de sólidos solubles y porcentaje de materia seca siendo significativamente mayor en los frutos cultivados bajo malla antiinsectos (T1), lo que coincide con diversos autores (Rosales et al., 2008; Max et al., 2009; Rosales et al., 2009) y puede atribuirse al efecto de algunas condiciones de estrés en las plantas que están bajo cubierta de malla antiinsectos, por un menor control sobre las condiciones climáticas de estos invernaderos respecto a uno de mayor tecnología.

\section{CONCLUSIÓN}

Los resultados de este trabajo no mostraron tendencias a diferencias significativas en los parámetros de fertirriego ni en la producción total al cultivar en un invernadero de multitúnel de alta tecnología o en un invernadero de baja tecnología cubierto con malla antiinsectos. Respecto a la calidad de frutos, tanto los sólidos solubles y como el porcentaje de materia seca aumentan al cultivar en un invernadero de baja tecnología. Esto sugiere que el cultivo de tomate cherry es sensible a las condiciones ambientales que se producen al interior del invernadero.

Cuadro 3

Producción y calidad de frutos de tomate cherry, según invernadero: alta tecnología (T0) y baja tecnología (T1)

\begin{tabular}{|c|c|c|c|c|c|}
\cline { 2 - 6 } \multicolumn{1}{c|}{} & \multicolumn{2}{c|}{ Producción } & \multicolumn{3}{c|}{ Calidad de frutos } \\
\cline { 2 - 6 } \multicolumn{1}{c|}{} & $\mathbf{K g ~ m}^{-\mathbf{1}}$ & $\mathbf{N}^{\mathbf{0}}$ frutos $\mathbf{~ m}^{-\mathbf{1}}$ & $\mathbf{S S}$ ( $\left.{ }^{\mathbf{}} \mathbf{B r i x}\right)$ & $\mathbf{F F}(\mathbf{k g})$ & \% $\mathbf{~ M S}$ \\
\hline $\mathrm{T} 0$ & 3,79 & 342 & 8,43 & 1,11 & 8,47 \\
\hline $\mathrm{T} 1$ & 3,24 & 293 & 9,20 & 1,14 & 9,67 \\
\hline$p$ & $\mathrm{~ns}$ & $\mathrm{~ns}$ & $*$ & $\mathrm{~ns}$ & $* *$ \\
\hline
\end{tabular}

$*$, **, ***, ns, son $P \leq 0,05, P \leq 0,01, P \leq 0,001$ y no significativo, respectivamente.

SS: Sólidos solubles; FF: Firmeza de fruto; MS: Materia seca. 
Considerando los resultados de esta investigación se concluye que, bajo las condiciones de este trabajo, el cultivo en malla antiinsectos no afecta la producción y mejora la calidad de los frutos de tomate cherry.

\section{LITERATURA CITADA}

ABDUL-BAKI, A.A.; STOMMEL, J.R. 1995. Pollen viability and fruit set of tomato genotypes under optimum and high temperature regimes. HortScience 30, 115-117.

ADAMS, P.; HO, L.C. 1993. Effects of environment on the uptake and distribution of calcium in tomato and on the incidence of blossom-end rot. Plant Soil 154, 127-132.

HARMANTO, TANTAU, H.J.; SALONKE, V.M. 2006. Microclimate and air exchange rates in greenhouses covered with different nets in the humid tropics. Biosyst. Eng. 94, 239-253.

INSTITUTO NACIONAL DE ESTADÍSTICAS. 2008. VII Censo Agropecuario. Santiago, Chile.

LITTLE, T.M.; HILLS, F.J. 1976. Métodos estadísticos para la investigación en la agricultura. Ed. Trillas, México, pp. 270.

MAX, J.F.J.; HORST, W.J.; MUTWIWA, U.N.; TANTAU, H.-J. 2009. Effects of greenhouse cooling method on growth, fruit yield and quality of tomato (Solanum lycopersicum L.) in a tropical climate. Sci. Hortic 122, 179-186.

MÖLLER, M.; TANNY, J.; LI, Y.; COHEN, S. 2004. Measuring and predicting evapotranspiration in an insect-proof screenhouse. Agric. Forest Meteorol. 127, 35-51.

PEET, M.; SATO, S.; CLÉMENTE, C.; PRESSMAN, E. 2003. Heat stress increases sensitivity of pollen, fruit and

\section{AGRADECIMIENTOS}

Este trabajo fue financiado por el proyecto UTA 9720-08. seed production in tomatoes (Lycopersicon esculentum Mill.) to non-optimal vapour pressure deficits. Acta Hortic. 618, 209-215.

PETERSEN, R.G. 1994. Agricultural field experiments. Marcel Dekker Inc., New York, pp. 409.

ROMERO-GÓMEZ, P.; CHOI, C.Y.; LÓPEZ-CRUZ, I 2010. Enhancement of the greenhouse air ventilation rate under climate conditions of central México. Agrociencia 44, 1-15.

ROSALES, M.A.; CERVILLA, L.M.; RÍOS, J.J.; BLASCO, B.; SÁNCHEZ-RODRÍGUEZ, E.; ROMERO, L.; RUIZ, J.M. 2009. Environmental conditions affect pectin solubilization in cherry tomato fruits grown in two experimental Mediterranean greenhouses. Environ. Exp. Bot. 67, 320-327.

ROSALES, M.A.; RÍOS, J.J.; CERVILLA, L.M.; RUBIOWILHELMI, M.M.; BLASCO, B.; RUIZ, J.M.; ROMERO, L. 2008. Environmental conditions in relation to stress in cherry tomato fruits in two experimental Mediterranean greenhouses. J.Sci. Food Agric. 89,735-742.

SATO, S.; PEET, M.; THOMAS, J.F. 2000. Physiological factors limit fruit set of tomato (Lycopersicon esculentum Mill.) under chronic, mild heat stress. Plan Cell Environ. $23,719-726$. 